\title{
A STUDY OF INSULAR SEGMENT OF MIDDLE CEREBRAL ARTERY IN NORTHERN INDIA
}

\author{
Medha Das ${ }^{1}$ Pranjal Pankaj², Shirin Jahan ${ }^{3}$ \\ ${ }^{1}$ Associate Professor, Department of Anatomy, Rama Medical College, Mandhana, Kanpur. \\ ${ }^{2}$ Associate Professor, Department of Medicine, Rama Medical College, Mandhana, Kanpur. \\ 3Professor, Department of Anatomy, Rama Medical College, Mandhana, Kanpur.
}

\begin{abstract}
The microsurgical anatomy of middle cerebral artery is of particular interest to the cerebrovascular surgeons as it supplies most of the superolateral surface of cerebral hemispheres and is the most commonly involved artery in stroke. The insular segment (M2 segment) begins at the limen insulae and runs on the surface of the insula in the sylvian insular cistern with a superoposterior direction. The M2 segment consists of two or three branches that arise from the bifurcation or trifurcation of the M1. After reaching the top of the insula, these branches turn inferolaterally and exit from the sylvian insular cistern forming the M3 segment.
\end{abstract}

\section{OBJECTIVE}

Certain clinical conditions like aneurysms and glioma of the M2 segment aneurysms demands special attention due to vascular complexity of the insular area and peculiar clinical characteristics. The present study was carried out for a better understanding and to define further the microsurgical anatomy of the insular segment of middle cerebral artery hoping to find immediate application of our findings in the field of microsurgical cerebral revascularisation and better interpretation of radiological angiographic investigations performed in cases of young cerebral haemorrhages. The present study also elaborates the directly observed dissection findings rather than the other studies, which are mostly based on radiological findings.

\section{MATERIALS AND METHODS}

Total 20 Middle Cerebral Arteries (MCA) were studied obtained from 10 brains. Meticulous dissection was done and middle cerebral artery and its branches were exposed and cleaned in lateral sulcus on the inferior surface of brain. Digital photographs were taken. The number of samples was based on the availability of cadavers in the mentioned institute during the time of study.

\section{RESULT}

In all 20 MCAs, bifurcation was noted. In 15 out of 20 specimens, more than one major cortical branch was given by M1 segment before its division into secondary trunks at insula. No trifurcation was found.

\section{CONCLUSION}

Some structural and statistical variations were noted in our study as in origin of major cortical branches before bifurcation in $75 \%$ of cases and this knowledge will be helpful to neurosurgeons operating in insular area.

\section{KEYWORDS}

Middle Cerebral Artery, M1 Segment, M2 Segment, Cerebral Revascularisation.

HOW TO CITE THIS ARTICLE: Das M, Pankaj P, Jahan S. A study of insular segment of middle cerebral artery in northern India. J. Evolution Med. Dent. Sci. 2016;5(64):4582-4587, DOI: 10.14260/jemds/2016/1044

\section{INTRODUCTION}

The Middle Cerebral Artery (MCA) is one of the three major paired arteries that supplies blood to the cerebrum. The MCA arises from the internal carotid and continues into the lateral sulcus where it branches and projects to many parts of the lateral cerebral cortex. It also supplies blood to the anterior temporal lobes and the insular cortices.[1]

The left and right MCAs arise from bifurcations of the internal carotid arteries and thus are connected to the anterior cerebral arteries and the posterior communicating arteries, which connect to the posterior cerebral arteries. The MCAs are not considered a part of the circle of Willis. The middle cerebral artery can be classified into 4 parts.[2]

Financial or Other, Competing Interest: None.

Submission 22-06-2016, Peer Review 15-07-2016,

Acceptance 22-07-2016, Published 11-08-2016.

Corresponding Author:

Dr. Medha Das

Associate Professor,

Department of Anatomy,

Rama Medical College, Mandhana,

Kanpur-209217, Uttar Pradesh.

E-mail:drmedhadas@rediffmail.com

DOI: $10.14260 /$ jemds/2016/1044
- M1: The sphenoidal segment, so named due to its origin and loose lateral tracking of the sphenoid bone. Although, known as the horizontal segment, the segment may descend, remain flat, or extend posteriorly in different individuals. The M1 segment perforates the brain with numerous anterolateral central (Lateral lenticulostriate) arteries, which irrigate the basal ganglia.

- M2: Extending anteriorly on the insula, this segment is known as the insular segment. It is also known as the Sylvian segment when the opercular segments are included. The MCA branches may bifurcate or sometimes trifurcate into trunks in this segment, which then extend into branches that terminate towards the cortex.

- M3: The opercular segment extends laterally, exteriorly from the insula towards the cortex. This segment is sometimes grouped as part of M2.

- M4: These finer terminal or cortical segments irrigate the cortex. They begin at the external end of the Sylvian fissure and extend distally away on the cortex of the brain. The M1 ends at the limen insulae where it sharply turns superiorly and posteriorly and divide to form the M2 segment. 
The M1 divides distally into two or sometimes three division (Bifurcation or trifurcation). In case of bifurcation, the divisions are superior and inferior trunks. In case of trifurcation, the additional division is middle trunk. Bifurcations and trifurcations occurs in $50 \%$ and $25 \%$ of the cases respectively.[3]

The cortical branches of the middle cerebral artery are described by the areas they irrigate.[4-5]

\section{BRANCHES SUPPLYING FRONTAL LOBE}

- Orbitofrontal Artery (OBFA): This branches out anterior, superior, and laterally to vascularise the inferior frontal gyrus. This "competes" in size with the frontal polar branch of the anterior cerebral artery.

- Prefrontal Arteries (PFA): The arteries fan out over the insula and exit to the cortex via the medial surface of the frontal operculum. The arteries fan superiorly over the pars triangularis and vascularises the inferior and middle frontal gyrus. Near the superior frontal gyrus, these arteries anastomose with branches from the pericallosal artery of the anterior cerebral artery.

- Precentral Artery (PCA): The artery extends out on the medial surface of the operculum and supplies the posterior parts of the middle and inferior frontal gyri as well as the lower parts of the precentral gyrus. This artery branches once or twice and is relatively invariant across anatomies.

- Central Arteries (CA): The artery extends out and exits from the central portion of the operculum then passing inside the central sulcus. This artery bifurcates in $72 \%$ of individuals and irrigate the posterior precentral gyrus and the inferior portion of the post-central gyrus.

\section{BRANCHES SUPPLYING PARIETAL LOBE}

- Anterior Parietal Artery (APA): This artery usually originates from the anterior or middle MCA trunk. In some cases, it branches from the rolandic artery or from the posterior parietal artery. Extends the interparietal sulcus and descends slightly posteriorly.

- Posterior Parietal Artery (PPA): Emerging from the posterior end of the Sylvian fissure and extends first posteriorly and then anteriorly along the posterior of the parietal lobe. It also branches to the supramarginal gyrus.

- Angular Artery: The angular artery is a significant terminal branch of the anterior or middle trunk of the MCA. It emerges from the Sylvian fissure and passes over the anterior transverse temporal gyrus and usually divides into two branches. One of the branches supply the angular gyrus while the other supplies the supramarginal gyrus, posterior superior temporal gyrus, and the parietooccipital arcus (Sulcus).

- Temporal Occipital Artery: The longest cortical artery, it run posteriorly and opposite to operculum centre upon its exit from the Sylvian fissure. It run parallel to the superior temporal sulcus and supplies the superior and inferior occipital gyri. This vessel anastomoses with the posterior cerebral artery and may exist as one or two arteries, $67 \%$ or $33 \%$ of the time, respectively.

\section{BRANCHES SUPPLYING TEMPORAL LOBE}

- Temporopolar Artery (TPA): The artery extends from the sphenoidal segment of the MCA via the operculum inferior surface and supplies the polar and anterior lateral portions of the temporal lobe. The vessel can be identified in $52 \%$ of normal angiograms.

- $\quad$ Anterior Temporal Artery (ATA): This artery extends in the similar fashion and the temporopolar and vascularises the same regions.

- Middle Temporal Artery (MTA): This artery extends from the Sylvian fissure opposite to the inferior frontal gyrus and supplies the superior and middle portion of the middle temporal lobe. It can be identified in $79 \%$ of angiograms.

- $\quad$ Posterior Temporal Artery (PTA): This artery extends out and away from the operculum and turns in a step-wise manner first inferiorly, then posteriorly into the superior temporal sulcus, then to the middle temporal sulcus. This vessel supplies the posterior portion of the temporal lobe and is the origin of several perforating arteries that irrigate the insula. It is readily identifiable most radiograms.

MCA aneurysms account for approximately $20 \%$ of all intracranial aneurysms. ${ }^{[6,7]}$ MCA aneurysms has certain special features, which are not seen with aneurysms at other sites. Giant MCA aneurysms are more common than giant aneurysms in other locations with the possible exception of the paraclinoid region of the ICA.[8] This observation might relate to the fact that MCA aneurysms can reach massive proportions without producing symptoms from encroachment on vital structures. When large enough, unruptured MCA aneurysms can produce clinical symptoms from mass effect.[9] Temporal lobe epilepsy is another symptom that occurs rarely as a result of MCA aneurysms, but only exceptionally as a result of aneurysms in other locations. Ischaemic symptoms such as transient ischaemic attacks and small strokes, although rarely caused by aneurysms occur more frequently with aneurysms of the MCA than with aneurysms in other locations. Association between insular injury and autonomic derangements has been studied extensively in patients with ischaemic stroke and there seems to be hemispheric laterality in the autonomic derangements.[10,11] The Middle Cerebral Artery (MCA) bifurcation aneurysms are anatomically unique because they are located adjacent to the insula. Ruptured MCA aneurysms frequently cause Temporal Haematomas (THs) and resultant insular injury may cause autonomic derangements.

The insular lobe is a functionally complex structure harbouring peculiar anatomical and vascular features and specific neuronal connectivity with surrounding cerebral structures. It is situated in the depth of the Sylvian fissure and can be affected by either low-grade or high-grade gliomas. Because of its complexity, surgery of insular tumours has been traditionally regarded as hazardous. Nonetheless, currently improved diagnostic, neurophysiological, and surgical tool allow the neurosurgeon to perform surgery of insular gliomas in a safer way. The aim of this paper is to provide the reader with an updated review of the anatomy of the insular segment of MCA for better understanding of the regional vascular anatomy and safer surgeries. 


\section{MATERIALS AND METHODS}

The present work was carried out in Department of Anatomy, RIMS, Ranchi and continued in Rama Medical College, Kanpur. Middle cerebral artery was observed in the brain obtained from cadaver used for routine educational dissection. The brain was removed by dissection method mentioned in Cunningham's Manual of Practical Anatomy.[12] Total 20 MCAs were studied obtained from 10 brains.

The number of samples was based on the availability of cadavers in the mentioned institute during the time of study. After removal of brain from the skull, blood vessels on the base of brain were cleaned from pia mater on the external surface of which it lies. Through meticulous dissection middle cerebral artery was cleaned. The division of the main trunk and the secondary trunks was studied and documented. The secondary trunks are referred to as superior, middle (If present), and inferior trunks depending on the way in which the main trunk of the MCA is divided.

\section{Type of Study}

Descriptive observational study.

\section{Inclusion Criteria}

Undamaged specimens of the brain with intact middle cerebral artery.

\section{Exclusion Criteria}

Damaged middle cerebral artery.

\section{OBSERVATION}

In all the specimens, Middle Cerebral Artery (MCA) originated from Internal Carotid Artery (ICA) as the larger terminal branch opposite optic chiasma. The major cortical branches were seen to arise from M1 segment of all MCAs before its division into secondary trunks in the insula. In $75 \%$ of specimens (15 out of 20), more than one major cortical branch was given by M1 segment. These branches were orbitofrontal artery and temporal branches. (Fig. 6).

\begin{tabular}{|c|c|c|c|c|}
\hline Branch & $\begin{array}{c}\text { Superior } \\
\text { Div. }\end{array}$ & $\begin{array}{c}\text { Inferior } \\
\text { Div. }\end{array}$ & M1 segment & Total \\
\hline OBFA & 5 & - & 15 & 20 \\
\hline PFA & $\begin{array}{c}16(2 \\
\text { from } \\
\text { OBFA })\end{array}$ & - & $\begin{array}{c}4(3 \text { from } \\
\text { OBFA })\end{array}$ & 20 \\
\hline PCA & 19 & - & $\begin{array}{c}1 \text { (direct } \\
\text { branch of } \\
\text { OBFA })\end{array}$ & 20 \\
\hline CA & 20 & - & - & 20 \\
\hline APA & 20 & - & - & 20 \\
\hline PPA & 20 & - & - & 20 \\
\hline TPA & - & - & 20 & 20 \\
\hline ATA & - & 4 & 16 & 20 \\
\hline MTA & - & 18 & 2 & 20 \\
\hline PTA & - & 18 & 2 & 20 \\
\hline $\begin{array}{c}\text { Angular } \\
\text { artery }\end{array}$ & 18 & - & 2 & 20 \\
\hline Table 1: Showing Origin of Cortical Branches of MCA \\
from Secondary Divisions (M2 Segment) \\
\hline \multicolumn{2}{|l}{}
\end{tabular}

In all 20 MCAs, bifurcation was noted, so the secondary trunks were superior division and inferior division (Fig. 1). No trifurcation was found though three cases of early pseudotrifurcation and two case of early pseudobifurcation was found.

In one brain on right side, thick polar temporal and anterior temporal artery was arising from M1 segment side by side giving appearance of early pseudotrifurcation. (Fig. 4).

In one brain on left side, orbitofrontal artery and prefrontal artery were seen to take origin from M1 segment side by side giving appearance of early division of MCA (Early pseudotrifurcation).

In one specimen on left side, the stem of origin of orbitofrontal artery from M1 segment was very thick giving appearance of early division of MCA. (Early pseudobifurcation). (Fig. 5).

In one brain on left side, orbitofrontal artery was very thick taking origin from M1 segment, which after giving the orbital branch continued and gave prefrontal and precentral artery, which are usually branches of superior division (M2 segment) giving appearance of early pseudobifurcation.

Orbitofrontal artery, which is generally a branch of superior division of M2 segment was found to originate from M1 segment in 15 out of 20 specimens. Precentral and prefrontal artery were also seen taking origin from orbitofrontal artery instead of usual origin from superior division in 1 and 5 specimens respectively. (Fig. 2).

Among the temporal branches, temporopolar artery was taking origin from M1 segment in all 20 specimens. Anterior temporal artery was originating from M1 segment in 16 out of 20 cases. Middle and posterior temporal artery was mostly taking origin from inferior division (18 out of 20). The temporal occipital artery when present was always a branch of inferior division. (Fig. 3). The origin of various cortical branches from secondary trunks of M2 segment of MCA is summarised in table.

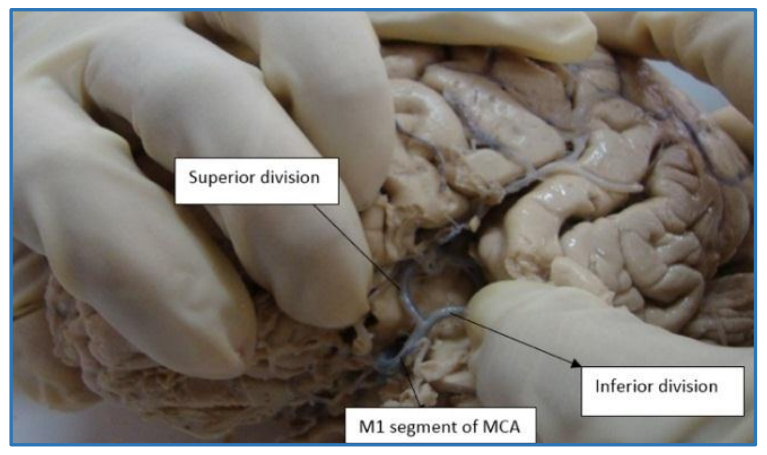

Fig. 1: Showing Bifurcation of Middle Cerebral Artery (MCA) at Insula

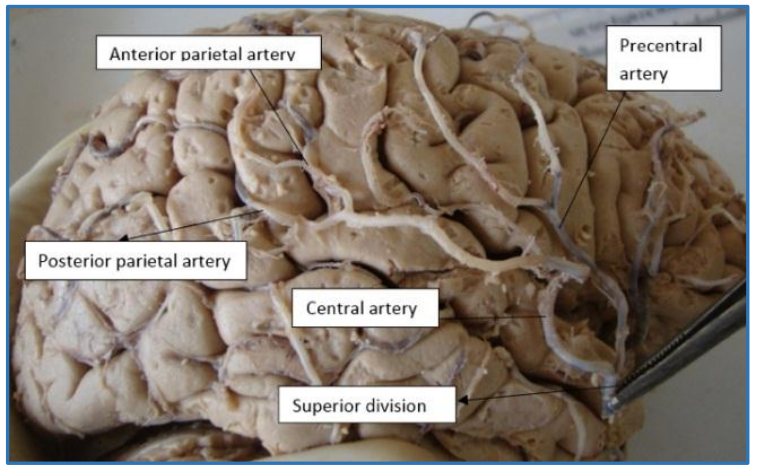

Fig. 2: Showing Cortical Branches Arising from Superior Division of M2 Segment of MCA 


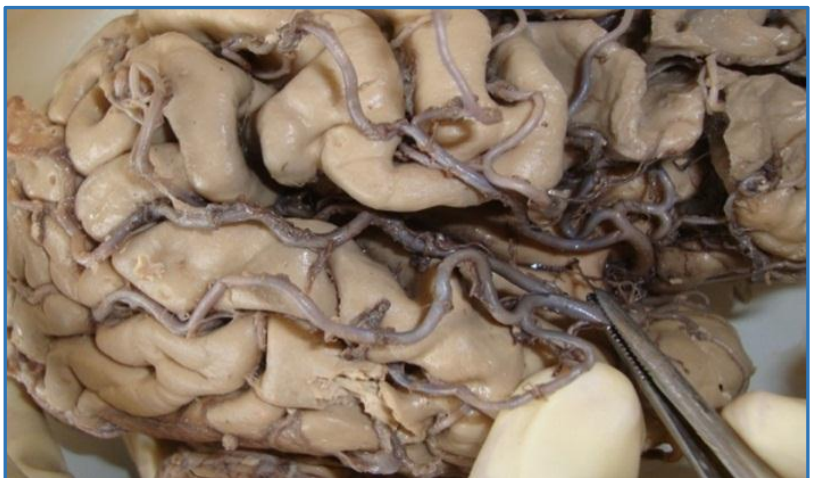

Fig. 3: Showing Branches from Inferior Division (Held with Forceps) of M2 Segment of Middle Cerebral Artery

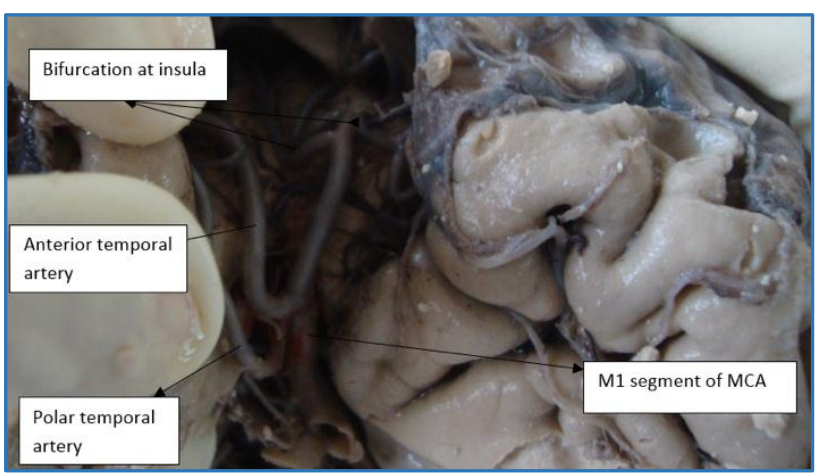

Fig. 4: Showing Thick Anterior Temporal and Polar Temporal Artery Arising Side by Side from M1 Segment of MCA on Right Side (Early Pseudotrifurcation)

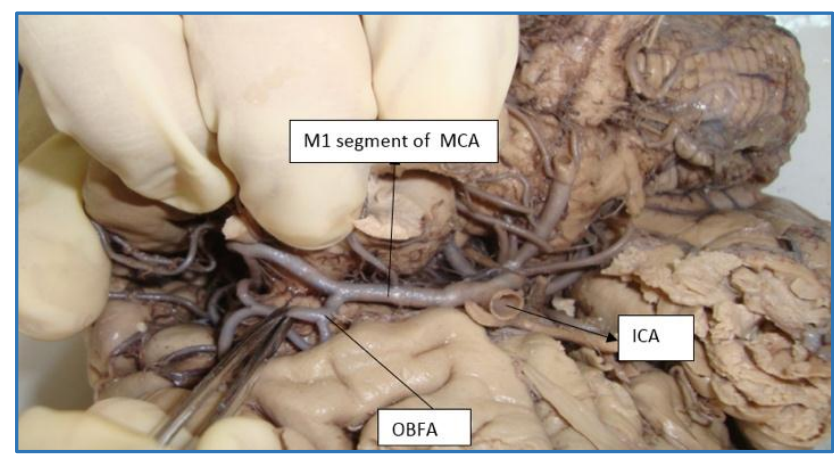

Fig. 5: Thick Orbitofrontal Artery (OBFA) Taking Origin from M1 Segment of MCA on Left Side (Early Pseudobifurcation). ICA = Internal Carotid Artery

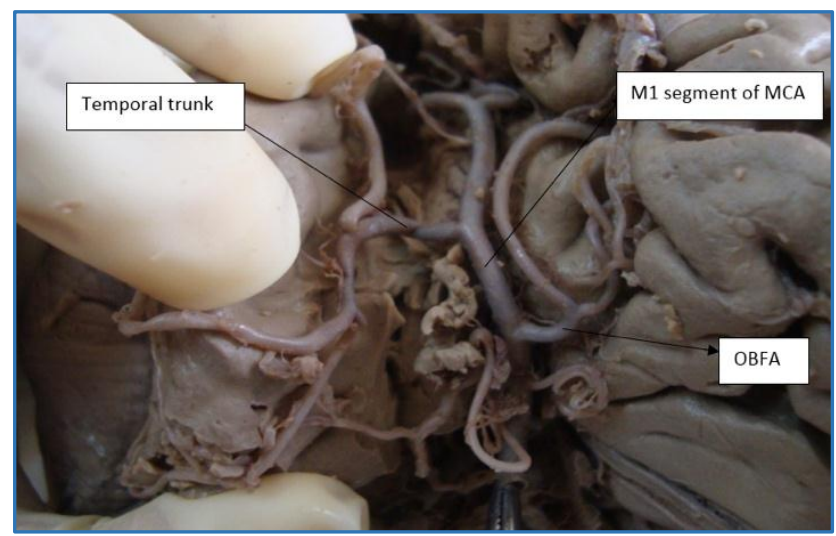

Fig. 6: Showing Origin of Two Major Cortical Branch (OBFA and Temporal Trunk) from MCA before Bifurcation. OBFA = Orbitofrontal Artery

\section{DISCUSSION}

The microsurgical anatomy of the middle cerebral artery has interested many researchers like Chater et al (1976), Grand (1980), Gibo et al (1981), Umansky et al (1984), and Yasargil over the years especially with the advent of microneurosurgical techniques in cerebrovascular surgery.[13] It supplies the largest area with the most complex branching of any of the intracerebral vessels.

Ring and Waddington have described the terminal configuration of the middle cerebral artery as well as the branching pattern of the artery within the Sylvian fissure. Ring (1962) studied the branching pattern of middle cerebral artery in 75 brain specimens. Out of these, study on 25 brains was dissection based. He described ten distinct forms of division. In 34\% specimens, one major vessel was arising proximal to sylvian fissure. In $26 \%$, more than one major vessel was arising proximal to fissure. In 24\%, trifurcation at the mouth of the fissure and in $16 \%$ bifurcation at the mouth of the fissure was documented. In the majority of cases, one or more vessels arose proximal to the fissure and the division of the remaining trunk in the mouth of the fissure was made up of somewhat irregular branching and rebranching without any constant pattern.[14-16]

On the basis of anatomical and angiographic studies, Michotey et al. (1974) and Salamon and Huang (1976) described two basic patterns that can help in identifying these arteries. In cases of bifurcation of the M1, two trunks are present: one anterior, the other posterior. The orbitofrontal, operculofrontal, and central arteries arise from the anterior trunk. The remaining branches - anterior and posterior parietal, gyrus angularis, and temporal arteries arise from the posterior trunk. In cases of trifurcation, the orbitofrontal and operculofrontal arise from the anterior trunk; the central, parietal, and gyrus angular arteries arise from the middle trunk; the temporal branches arise from the posterior trunk. $[17,18]$

Umansky studied the microvascular anatomy of the proximal segments (M1 and M2) of the middle cerebral artery (MCA) in 70 unfixed brain hemispheres from 35 cadavers. He divided MCA into four groups: Group I: absence of a main division (that is, a single-trunk type of MCA) (in $6 \%$ of cases); Group II: bifurcation (64\%); Group III: trifurcation (29\%); and Group IV: quadrifurcation (1\%). In 1986, Yasargil et al and Umansky et al suggested that the exact microanatomy of this division can be ascertained only intraoperatively. The size of the secondary trunks depends on the number of cortical branches arising from it and consequently the area supplied.[19]

According to Umansky et al, there are 4 types of branching patterns: 1) cortical branches arise as collateral vessels from a single trunk that terminates in the angular artery (6\%); 2) the MCA divides into the superior (Frontal) and inferior (Temporal) trunks (64\%); 3) the MCA divides into the superior, middle, and inferior trunks (29\%); and 4) the MCA divides into 4 trunks (1\%). In the study reported by Gibo et al,(1) $78 \%$ of the MCAs divided into a bifurcation, $12 \%$ divided into a trifurcation, and $10 \%$ divided into multiple trunks. Yasargil.(2) states that when a large branch arises from the superior or inferior trunk just after the bifurcation, a false impression or "pseudotrifurcation" or "pseudoquadrification" takes place. According to him, even a dominant early branch 
from M1 segment can give a false impression of early bifurcation of MCA.

There is usually a dominance of either superior or inferior division. The superior division will always by definition be responsible for the frontal convexity. The inferior division will supply the temporal lobe in balance with temporal branches of the posterior cerebral artery. The parietal lobe is variable when supplied by branches originating from inferior division, inferior trunk is larger and therefore dominant. Similarly, when it is supplied by branches from superior division; superior division is dominant. Trifurcation may reflect a separate parietal lobe trunk or any sort of combination/permutation of the many MCA branches.

In $22.5 \%$ hemispheres dissected by Ture et al, the anterior and posterior parietal arteries have their origin in the superior M2 segment branch. They mainly supply the inferior frontal cortex, the frontal opercular cortex, and also the cortex in parietal and central sulcus areas. The inferior (Temporal) M2 segment branch is the main origin of the posterior and middle temporal arteries supplying mainly the middle and posterior temporal cortex and temporal occipital, angular, and posterior parietal regions.[20]

In 2005, Pai S B and Varma R G did dissection-based study on the microsurgical anatomy of the MCA. He stated that bifurcation of the MCA occurs at the high point of the limen insulae. The area of the bifurcation was described as forming an 'omega' pattern. There were eight bifurcations and two trifurcations among 10 MCAs, he studied. Both the trifurcations were seen in the same patient on opposite sides. The middle trunk divided into the angular, central, and anterior parietal branches on one side and posterior parietal and temporal occipital on the other side. In his study, the orbitofrontal, prefrontal, precentral, central, and anterior parietal arteries owed their origins to the superior trunk in most situations. Similarly, the posterior parietal, middle temporal, posterior temporal, temporal occipital, and angular arteries arise from the inferior trunk. In the two MCA trifurcations that were noted, the middle trunk gave rise to the posterior parietal and temporal occipital arteries (Inferior trunk branches) in one case and AP, central, and angular arteries (Superior trunk branches) in the other. Some of the cortical branches maybe absent and the adjacent branches may share the blood flow.[21]

Our study of insular segment of middle cerebral artery differs from other studies mainly in the pattern of division of main trunk into secondary trunks and in variations in origin of cortical branches from secondary trunks. Though trifurcation and even tetrafurcation is reported earlier in many studies, not a single case of true trifurcation was found in our study.

\section{CONCLUSION}

Documentation of microsurgical anatomy of the middle cerebral artery is not very common in Indian literature. Knowledge of its microsurgical anatomy is essential for a neurosurgeon operating upon cerebrovascular disorders as MCA is most commonly involved artery in stroke. Also, middle cerebral artery aneurysms form about $18.4 \%$ of all cerebral aneurysms. Although, the microsurgical anatomy of the middle cerebral artery in northern Indian population correlated with the findings in the western literature, some structural and statistical variations were noted in our study mainly the absence of trifurcation and origin of major cortical branches before bifurcation in $75 \%$ of cases.

\section{REFERENCES}

1. Standring S, Gray H. Arterial supply of brain. Gray's anatomy. 40th ed. Spain: Elsevier Ltd 2008:250.

2. Hugo K, Yaşargil MG, Huber P, et al. Cerebral angiography. New York: Thieme Medical Publishers Inc 1982:105-23.

3. Boris G, Bradac. Chapter cerebral angiography, normal anatomy, and vascular pathology. $1^{\text {st }}$ ed. Springer-Verlag Berlin Heidelberg 2011:57-65.

4. Osborn AG, Jacobs JM. Diagnostic cerebral angiography. Philadelphia: Lippincott Williams and Wilkins 1999:1434.

5. Kiernan JA, Barr ML. Blood supply of the central nervous system. In: Kiernan JA, ed. Barr's the human nervous system: an anatomical viewpoint. Philadelphia: Lippincott-Raven 1998:439-55.

6. Robinson RG. Ruptured aneurysms of the middle cerebral artery. J Neurosurg 1971;35(1):25-33.

7. Sundt TM, Kobayashi S, Fode NC, et al. Results and complications of surgical management of 809 intracranial aneurysms in 722 cases: related and unrelated to grade of patient, type of aneurysm, and timing of surgery. J Neurosurg 1982;56(6):753-65.

8. Heros RC, Kolluri S. Giant intracranial aneurysms presenting with massive cerebral oedema. Neurosurgery 1984;15(4):572-7.

9. Hook O, Norlen G. Aneurysms of the middle cerebral artery. Acta Chir Scand 1958;235 Suppl:1-39.

10. Gibo H, Carver CC, Rhoton AL. Microsurgical anatomy of the middle cerebral artery. J Neurosurg 1981;54(2):15169.

11. Yasxargil MG. Operative anatomy of the middle cerebral artery. In: Yasxargil MG, ed. Microneurosurgery. Vol.1. Stuttgart: Thieme Verlag 1984:72-91.

12. Cunningham DJ, Romanes GJ. Cunningham's manual of practical anatomy: head and neck and brain. Oxford University Press 1971.

13. Umansky F, Juarez SM, Dujovny M, et al. Microsurgical anatomy of the proximal segments of the middle cerebral artery. J Neurosurg 1984;61(3):458-67.

14. Ring BA. Middle cerebral artery: anatomical and radiographic study. Acta Radiol 1962;57(4):289-300.

15. Ring BA, Waddington M. Ascending frontal branch of middle cerebral artery. Acta Radiol Diagn (Stockh) 1967;6(3):209-20.

16. Ring BA, Waddington M. The neglected cause of stroke: intracranial occlusion of the small arteries. Radiology 1967;88(5):924-9.

17. Michotey P, Grisoli F, Raybaud $\mathrm{CH}$, et al. Etude anatomique et radiologique de l'artere cerebrale moyenne. Procede de rerperage. Ann Radiol 1974;17:721.

18. Salamon G, Huang YP, Michotey P, et al. Radiological Anatomy of Brain. Springer Verlag Berlin Heidelberg 1976:16-46.

19. Yasargil MG. Intracranial arteries. In: Yasargil MG, ed. Microneurosurgery Vol.1. New York: Thieme Medical Publishers Inc 1987:54-164. 
20. Ture U, Yasxargil MG, Al-Mefty 0 , et al. Arteries of the insula. J Neurosurg 2000;92(4):676-87.
21. Pai SB, Varma RG, Kulkarni RN. Microsurgical anatomy of middle cerebral artery. Neurol India 2005;53(2):186-90. 